\title{
Nuclear ING2 expression is reduced in osteosarcoma
}

\author{
XIAO-RUI HAN, XI-ZHUANG BAI, YU SUN and YAN YANG \\ Department of Joint Surgery and Sports Medicine, The First Affiliated Hospital of \\ China Medical University, Shenyang, Liaoning 110001, P.R. China
}

Received June 11, 2014; Accepted August 5, 2014

DOI: $10.3892 / o r .2014 .3458$

\begin{abstract}
Osteosarcoma is a high-grade malignant bone tumor. Loss of inhibitor of growth 2 (ING2) expression has been demonstrated in numerous types of cancers. However, no study has shown the relationship between ING2 expression and osteosarcoma. In the present study, we confirmed that the levels of ING2 mRNA and protein were lower in cancer tissues than these levels in normal tissues. Loss of nuclear ING2 protein was significantly associated with a decreased survival time of patients. Osteosarcoma cells were transfected with ING2 protein without a nuclear localization signal or intact ING2 protein to examine the effects of exogenous expression of ING2 in vitro. Compared to the control cells, intact ING2expressing cells exhibited increased apoptosis, G1 phase arrest and senescence. Taken together, these results suggest that ING2 acts as a tumor suppressor in osteosarcoma.
\end{abstract}

\section{Introduction}

Osteosarcoma is a primary tumor of the bone that accounts for $5 \%$ of all childhood cancers and represents the fifth most common tumor in young adults (1). It usually arises in the metaphysis of long bones such as the distal femur, proximal tibia and proximal humerus (2). Modified chemotherapy and surgical procedures, accompanied by the application of radiotherapy, have not led to any major improvements in the treatment outcome and quality of life of osteosarcoma patients (3). Therfore, it is urgent to further understand the mechanisms involved in the tumorigenesis of osteosarcoma in order to identify new therapeutic targets (4).

The inhibitor of growth (ING) family proteins are candidate tumor suppressors that associate with histone acetyltransferase (HAT), histone deacetylase and factor acetyltransferase complexes (5). Inhibitor of growth 2 (ING2) is the second member of the ING family of candidate tumor-suppressor

Correspondence to: Dr Xiao-Rui Han, Department of Joint Surgery and Sports Medicine, The First Affiliated Hospital of China Medical University, Shenyang, Liaoning 110001, P.R. China

E-mail: hanxr_2008@163.com

Key words: inhibitor of growth 2, osteosarcoma, cell cycle, apoptosis, senescence genes ING1 to ING5 (6-8). ING2 consists of three exons, exon $1 \mathrm{a}, 1 \mathrm{~b}$, and 2 , resulting in two transcribed isoforms: ING2a and ING2b (9). Many studies have found that ING2 expression is decreased or lost in human tumors, which suggests a role for ING2 as a tumor-suppressor gene. Borkosky et al (10) found that ING2 LOH may occur in late stages during head and neck squamous cell carcinoma progression. In addition, Zhang et al (11) found that ING2 expression was significantly decreased in hepatocellular carcinoma (HCC) and may lead to an unfavorable prognosis. However, in a study by Kumamoto et al (12), the authors confirmed that ING2 is upregulated in colon cancer. To our knowledge, no related study has demonstrated a relationship between ING2 and osteosarcoma. Our results could help in elucidating the functional role of ING2 in osteosarcoma.

\section{Materials and methods}

Tumor cells and osteosarcoma tissue samples. The human osteosarcoma cell line, HOS, was obtained from the American Tissue Type Collection (ATCC, Bethesda, MD, USA) and was maintained in minimum essential medium (MEM; Life Technologies, Gaithersburg, MD, USA) supplemented with $10 \%(\mathrm{v} / \mathrm{v})$ fetal bovine serum (FBS) and antibiotics $(100 \mathrm{U} / \mathrm{ml}$ of penicillin and $100 \mathrm{mg} / \mathrm{ml}$ of streptomycin) at $37^{\circ} \mathrm{C}$ in a $5 \%(\mathrm{v} / \mathrm{v}) \mathrm{CO}_{2}$ incubator. All the samples of osteosarcoma tissues and adjacent samples of non-tumorous tissues were obtained from the First Hospital of China Medical University from July 2003 to July 2013, following consent of each patient. The procedure was approved by the China Medical University Ethics Committee.

Real-time PCR. Total RNA was isolated using an RNeasy Mini kit (Biomed, Beijing, China). First-strand cDNA was reverse transcribed with $1 \mu \mathrm{g}$ of total RNA, using the Takara reverse transcription kit (Takara, Dalian, China) and oligo (dT)15 primers (Takara). The resultant cDNA was then used for quantitative PCR reactions. The ING2 primers were: 5'-GCGAGAGCTGGACAACAAAT-3' (sense) and 5'-GACACTTGGTTGCATAAGCAG-3' (antisense). The housekeeping gene, GAPDH, was used as an internal control for normalization of the results. The GAPDH primers were: 5'-AGAAGGCTGGGGCTCATTTG-3' (sense) and 5'-CGATCCACACGGAGTACTTGC-3' (antisense). Amplification of ING2 and GADPH was performed with 1 cycle at $95^{\circ} \mathrm{C}$ for $10 \mathrm{~min}$, and 40 cycles of $95^{\circ} \mathrm{C}$ for $15 \mathrm{sec}$ 
and $60^{\circ} \mathrm{C}$ for $60 \mathrm{sec}$. Calculation of the relative expression of each transcript was performed using the $2^{-\Delta \Delta C t}$ method.

Plasmids and transfection. The plasmids, pcDNA3.1-ING2 and pcDNA3.1-ING2- $\triangle$ NLS, were kindly provided by Mr. Xin-Yu Zhang (China Medical University, Shenyang). Transfection of the plasmids into HOS cells was performed using Lipofectamine ${ }^{\mathrm{TM}} 2000$ (Invitrogen Gibco, Carlsbad, CA, USA) according to the manufacturer's instructions. Cell lines transfected with pcDNA3.1 were used as a mock.

Western blot analysis. Nuclear and cytoplasmic proteins were isolated from the cells using the PARIS ${ }^{\mathrm{TM}}$ (Protein and RNA Isolation System) kit (Ambion Inc., Austin, TX, USA). Equal amounts $(30 \mu \mathrm{g})$ of cell lysates were separated by $10 \%$ SDS-polyacrylamide gel electrophoresis and transferred to polyvinylidene difluoride membranes, and incubated with the following specific antibodies. The ING2 antibody (sc-67646; Santa Cruz Biotechnology, Santa Cruz, CA, USA) was used to identify transfection efficiency. $\beta$-actin and $\beta$-tubulin (sc-130675 and sc-9104, respectively; both from Santa Cruz Biotechnology) were used as internal controls. The reaction was followed by probing with peroxidase-coupled secondary antibodies including anti-rabbit IgG or anti-mouse IgG at dilutions ranging from 1:1,000 to 1:2,000 (Amersham Biosciences, Needham, MA, USA), and the binding results were visualized by enhanced chemiluminescence (Amersham Pharmacia, Piscataway, NJ, USA).

Immunofluorescence. Cells were washed with PBS, fixed in $4 \%$ paraformaldehyde, permeabilized in $1 \%$ Triton X-100 for 5 min, and blocked with $5 \%$ bovine serum albumin in PBS containing $0.5 \%$ Triton X-100 for $1 \mathrm{~h}$. ING2 was detected using anti-ING2 (Santa Cruz Biotechnology) for $1 \mathrm{~h}$ at room temperature. Cells were washed with PBS and incubated with Alexa Fluor ${ }^{\circledast} 488$ donkey anti-goat $\operatorname{IgG}(\mathrm{H}+\mathrm{L})$ for $1 \mathrm{~h}$ at room temperature. Photographic images were captured using an Olympus CX71 fluorescence microscope (Olympus, Tokyo, Japan).

Physico-chemical profiles of ING2. Physico-chemical profiles, such as titration curve, hydrophobicity (13), antigenicity (14), fexibility (15), and solvent accessibility (16), were analyzed using Antheprot 5.0 software.

The PHYRE database was used to generate predicted structural models. The protein sequence of ING2 was obtained from Pubmed (http://www.ncbi.nlm.nih.gov/protein/AAQ13674.1) and submitted to Protein Homology/analogY Recognition Engine (PHYRE ver. 2). Based on the homology sequence in the PHYRE server, the three-dimensional structure of ING2 protein was predicted.

Cell cycle and apoptosis analyses. Cells were collected in PBS and fixed on ice with $1 \%$ paraformaldehyde, followed by $70 \%$ cold ethanol containing $10 \mu \mathrm{g} / \mathrm{ml}$ RNase. Then the cells were stained with $50 \mu \mathrm{g} / \mathrm{ml}$ propidium iodide (PI; KeyGen, Nanjing, China) for $15 \mathrm{~min}$ at room temperature for cell cycle analysis. The apoptotic cells were detected with Annexin V-FITC/PI double staining. Based on the manufac- turer's instructions for the apoptosis assay kit (KeyGen), the stained cells were analyzed by flow cytometry. Data analysis was performed with CellQuest software (BD Biosciences, Rockville, MD, USA).

SA- $\beta$-gal analysis. For SA- $\beta$-gal staining, cells were washed twice in PBS, fixed for 3-5 min at room temperature in 3\% formaldehyde and washed again with PBS. Then cells were incubated overnight at $37^{\circ} \mathrm{C}$ without $\mathrm{CO}_{2}$ in a freshly prepared SA- $\beta$-gal-staining solution as previously described (17).

DNA fiber spreading. Asynchronous cells were pulsed sequentially with $100 \mathrm{mM}$ BrdU (Zymed Laboratories, San Francisco, CA, USA) and $100 \mathrm{mM}$ IdU (Sigma Aldrich, St. Louis, MO, USA) for $20 \mathrm{~min}$ each. Cells were collected and DNA fibers were spread onto microscope slides as described by Jackson and Pombo (18).

Affymetrix GeneChip technology. The total RNA was extracted from the cells as described above. The total RNA samples were then analyzed by CapitalBio Corp., for GeneChip (Affymetrix, Santa Clara, CA, USA) assay, and each treatment had 3 biological replicates that were measured in this manner. Gene expression analysis was performed using the Affymetrix GeneChip, according to the laboratory methods in the Affymetrix GeneChip expression manual. Gene expression analysis was performed using triple arrays and triple independent mRNA samples for each treatment. Microarray data were analyzed using Bio MAS 3.0 software (CapitalBio, Beijing, China). Using the criterion of cutoff limitation as a fold change $\geq 2$ or $\leq 0.5$ and Q-value $\leq 5 \%$, differentially expressed genes were screened and clustered.

Statistics and survival analysis. Overall survival rates were determined using the Kaplan-Meier estimator. Kaplan-Meier survival plots were generated, and comparisons were made with log-rank statistics. For all analyses, a $\mathrm{P}$ value $<0.05$ was considered to indicate a statistically significant difference. All of the statistical analyses and graphics were performed with GraphPad Prism 5.

\section{Results}

Reduced ING2 nuclear expression in osteosarcoma. We examined ING2 expression in osteosarcoma by western blot analysis and real-time PCR. Results showed that the levels of ING2 mRNA and nuclear expression were decreased in tumor tissues relative to the levels in the normal tissues $(\mathrm{P}<0.05$, Fig. 1A and B). There was no significant difference in ING2 cytoplasmic expression between the normal tissues and tumor tissues ( $\mathrm{P}>0.05$, Fig. 1A). Follow-up information was available for the patients for periods ranging from 1 month to 5 years (median, 21 months). A Kaplan-Meier analysis showed that ING2 nuclear expression was closely correlated with the favorable prognosis of patients with osteosarcoma, whereas negative ING2 nuclear expression was correlated with a poor prognosis $(\mathrm{P}<0.05$, Fig. 1C).

ING2 expression in HOS cells. HOS cells were transfected with the pcDNA3.1-ING2 or pcDNA3.1-ING2- $\Delta$ NLS 
A
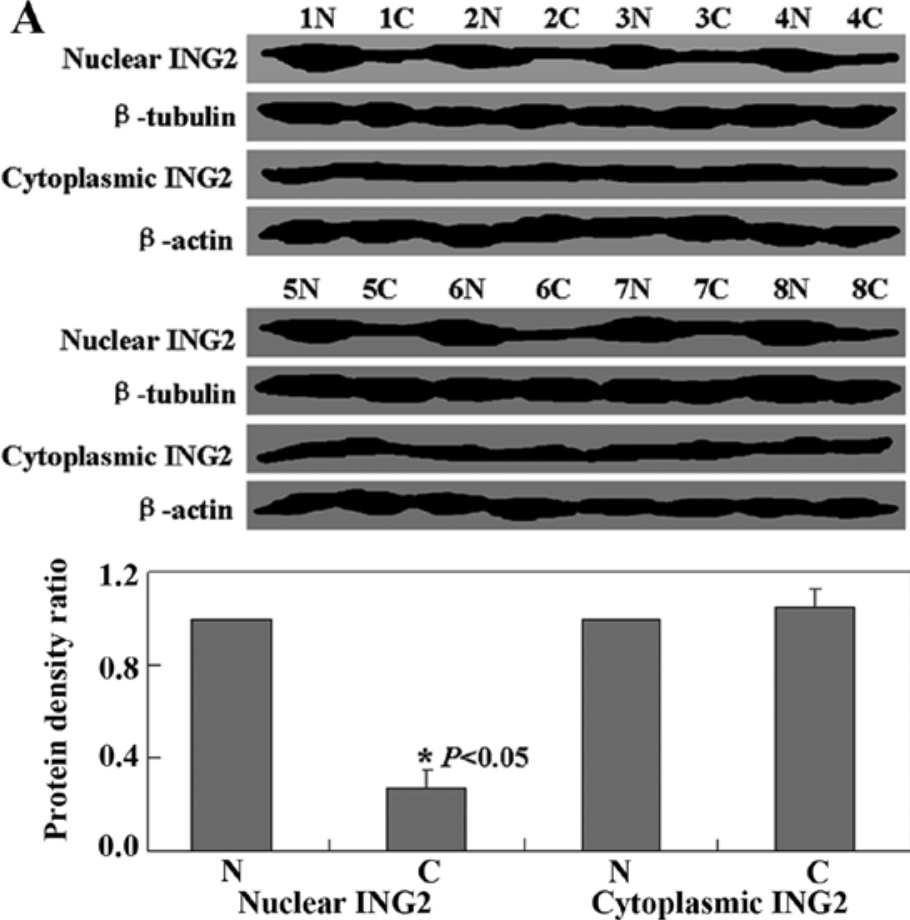

B
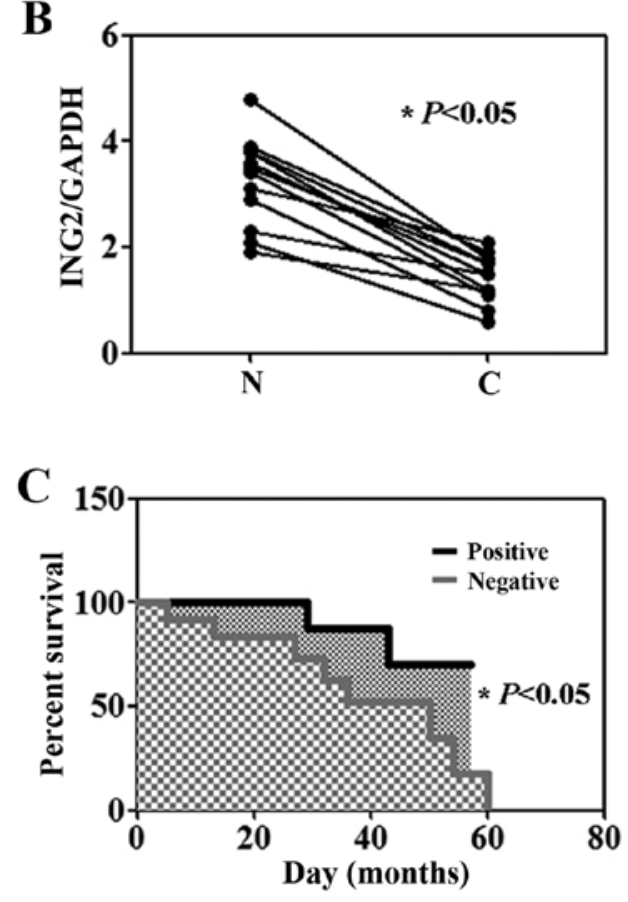

Figure 1. (A) Representative results of ING2 expression in osteosarcoma and corresponding normal tissues by western blot analysis. $\beta$-actin was used as an internal control. (B) The levels of ING2 mRNA were measured in specimens using real-time PCR. GAPDH was used as an internal control. (C) Kaplan-Meier curves of the cumulative survival rate of patients with osteosarcoma based on ING2 expression.

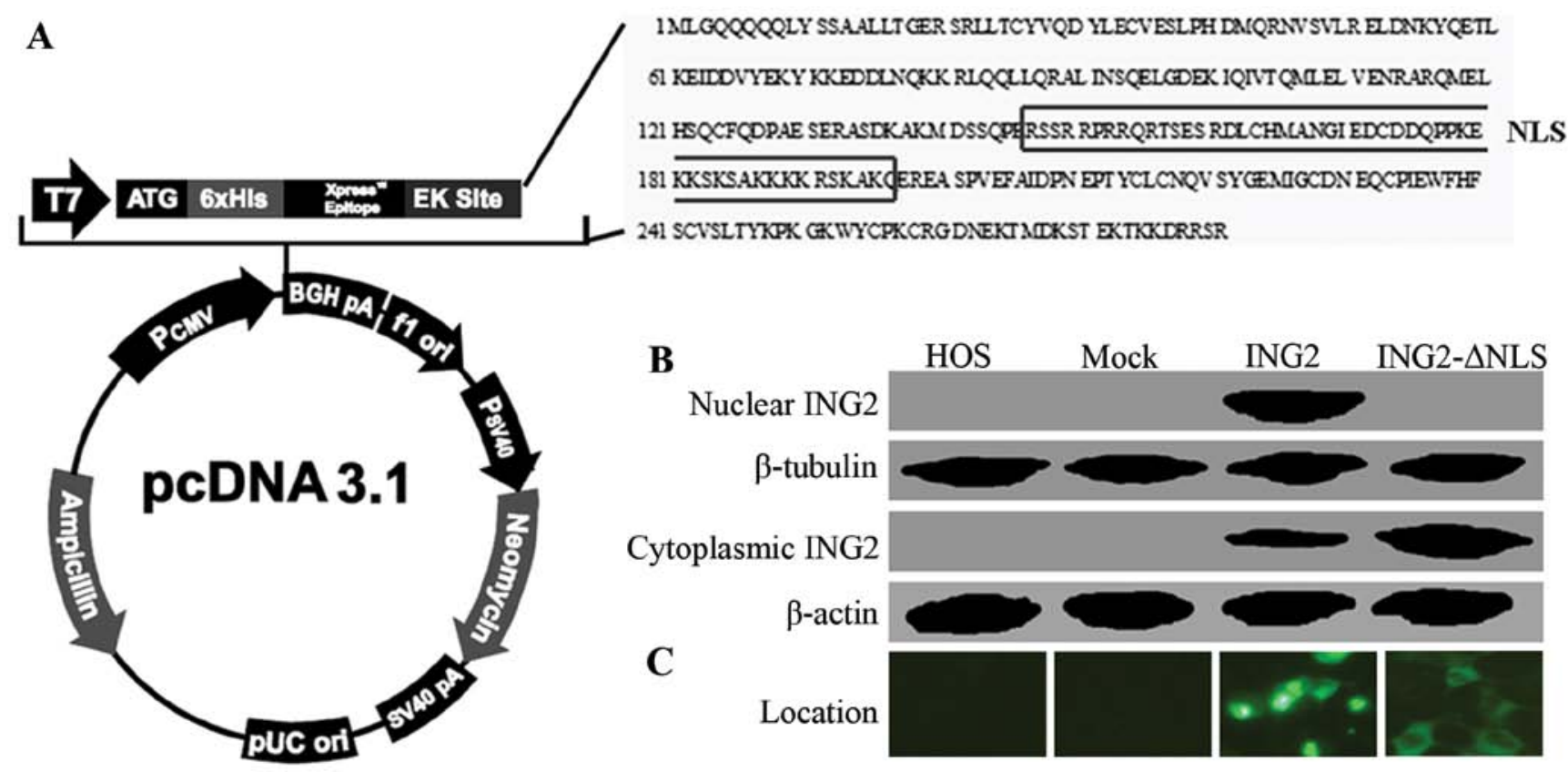

Figure 2. (A) Schematic diagram of pcDNA3.1-ING2 and pcDNA3.1-ING2- $\Delta$ NLS. (B) ING2 protein levels in HOS cells after transfection with pcDNA3.1-ING2 or pcDNA3.1-ING2- $\triangle$ NLS were detected using western blotting. (C) Detection of ING2 in the transfected and untransfected HOS cells by immunofluorescence.

expression vector. Schematic illustration of pcDNA3.1-ING2 and pcDNA3.1-ING2- $\Delta$ NLS are shown in Fig. 2A. The levels of ING2 cytoplasmic and nuclear expression were measured using western blotting (Fig. 2B). As shown in Fig. 2C, the results of immunofluorescence confirmed the location of ING2 in the HOS cells after transfection. Furthermore, titration curve, hydrophobicity, antigenicity, fexibility and solvent accessibility of the ING2 and ING2- $\Delta$ NLS proteins did not have a significant difference as assessed using Antheprot 5.0 software (Fig. 3A, B and D). Moreover, the 3D-structures of the two proteins were similar to each other (Fig. 3C).

Effect of ING2 expression on HOS cells in vitro. The ratio of the apoptotic cells present was determined using flow 

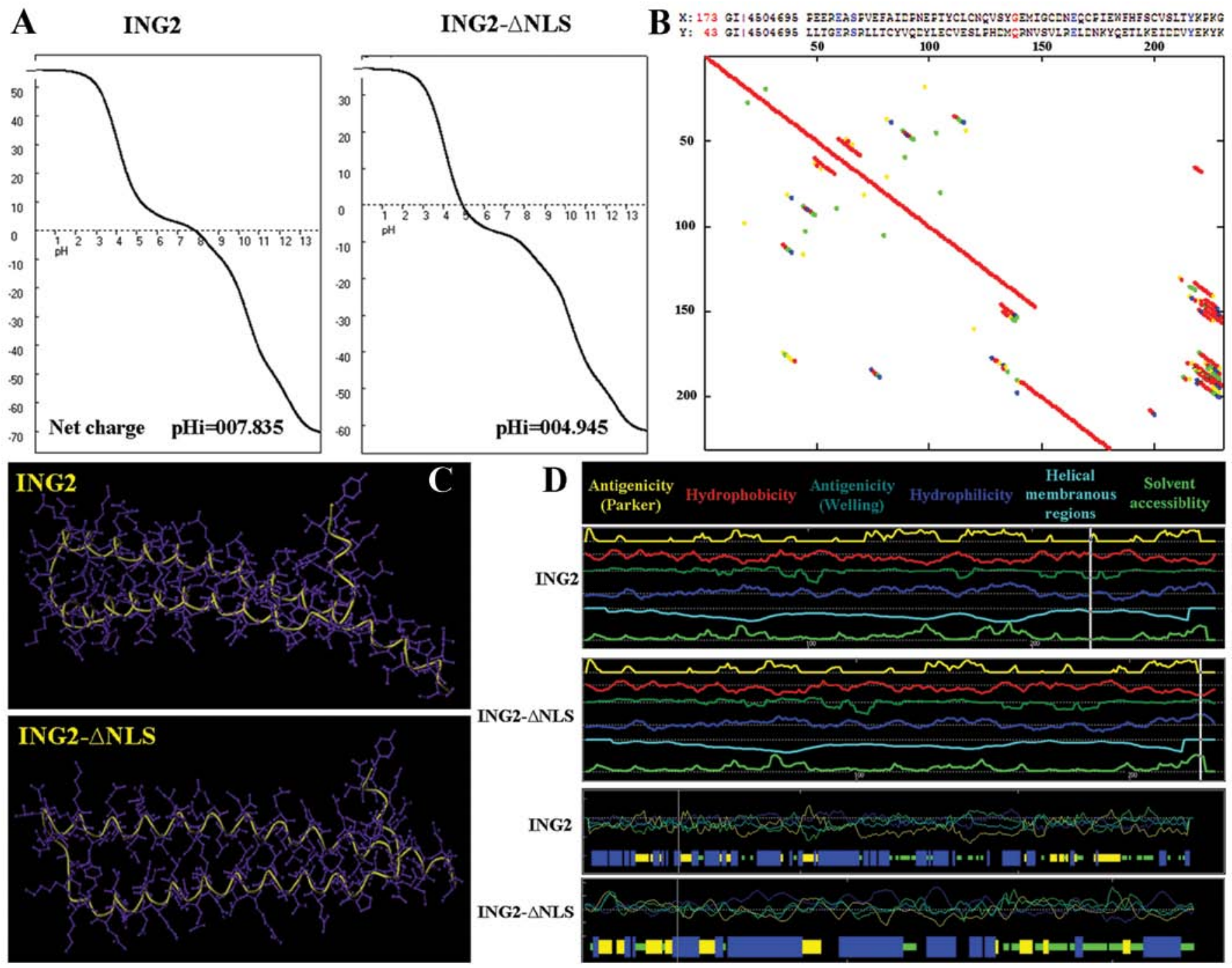

Figure 3. (A) Titration curves of ING2 protein and ING2- $\Delta$ NLS protein. (B) The dot-matrix plot of ING2 protein vs. ING2- $\Delta$ NLS protein. (C) $3 D$ structure of ING2 protein and ING2- $\Delta$ NLS protein. (D) Physico-chemical profiles of these two proteins.

cytometry. As shown in Fig. 4A, the percentage of apoptotic HOS cells in the pcDNA3.1-ING2-transfected group was 4- to 6-times higher than that in the untreated, mock or the pcDNA3.1-ING2- $\Delta$ NLS-transfected group $(\mathrm{P}<0.05)$. Cell cycle distribution of the transfected and untransfected cells was examined using PI staining. The ratio of cells in the G1 phase was found to be increased in cells in the pcDNA3.1-ING2transfected group when compared with the ratio in the other three groups $(\mathrm{P}<0.05$, Fig. 4B). Nearly all of the HOS cells in the pcDNA3.1-ING2-transfected group showed strong levels of blue SA- $\beta$-gal staining, while cells in the other three groups showed a lower frequency of $\mathrm{SA}-\beta$-gal staining $(\mathrm{P}<0.05$, Fig. 4C). Moreover, the results of the BrdU/IdU double labeling method showed that less HOS cells in the pcDNA3.1-ING2transfected group were in the duplicate phase when compared with the other three groups (Fig. 4D).

Affymetrix GeneChip analysis. Clustering of genes based on their relative expression in the HOS cells with ING2 expression compared with the untreated cells was carried out using the Affymetrix ${ }^{\circledR}$ human expression array (Fig. 5A). We arranged the networks of ING2 using Bio MAS (Molecule Annotation System) 3.0 software. However, many of these networks were consistent with the results that were predicted using Gene Ontology (GO) software (http://www.geneontology.org/) (Fig. 5B).

\section{Discussion}

The main purpose of the present study was to investigate the roles of the tumor suppressor ING2 in osteosarcoma. We for the first time demonstrated that ING2 nuclear expression was reduced in osteosarcoma. In previous studies, ING2 mRNA expression was found to be reduced in HCCs and in non-small cell lung carcinomas (19,20). Loss of ING2 nuclear expression has been reported in melanoma (21). Notably, in the present study, we found that ING2 was also expressed in the cytoplasm.

In order to fully understand the function of ING2 nuclear and cytoplasmic expression, osteosarcoma cells were transfected with an intact ING2 sequence or an ING2 sequence without a nuclear localization signal (NLS). Firstly, we compared the physico-chemical profiles of the nuclear and 
A

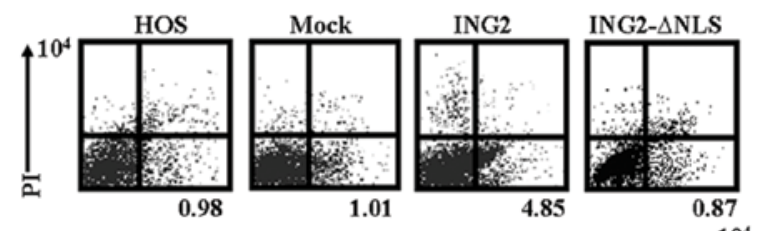

Annexin V-FITC

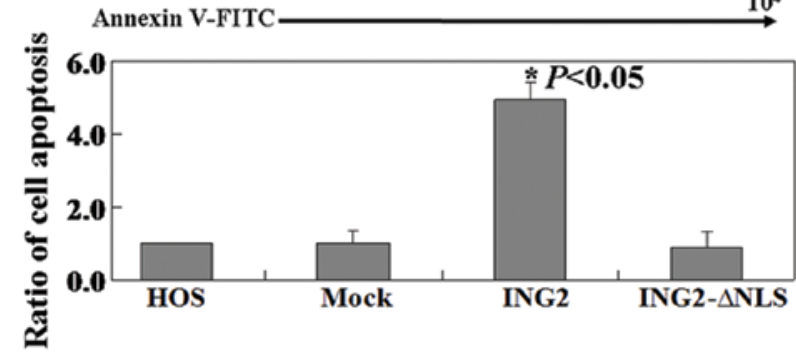

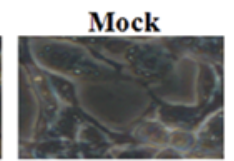
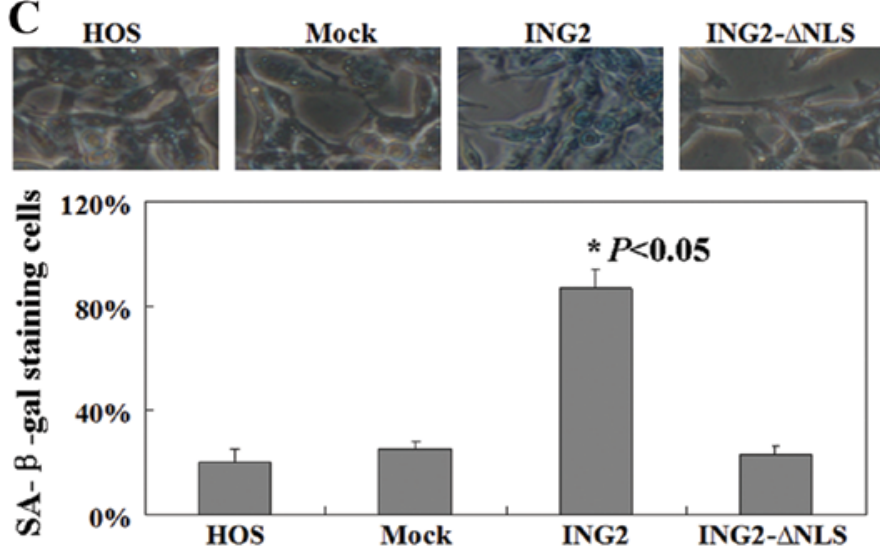

B
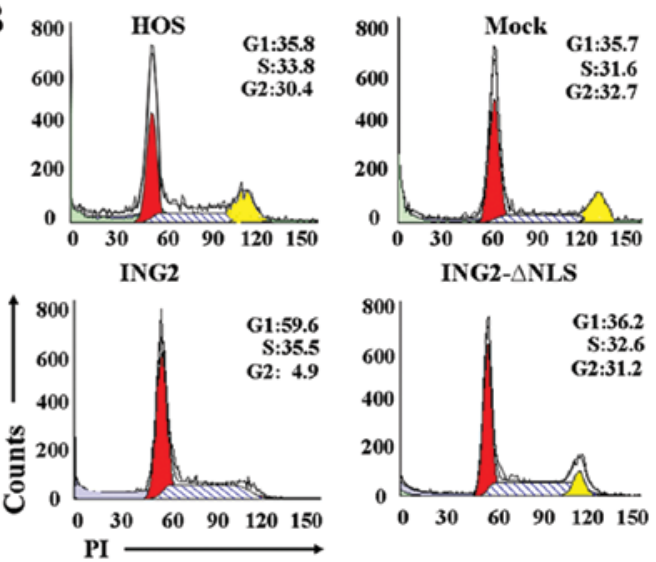

\section{Ongoing forks} Newly fired origins

Terminations
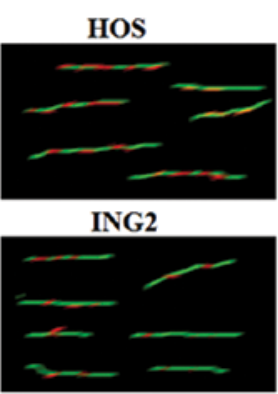

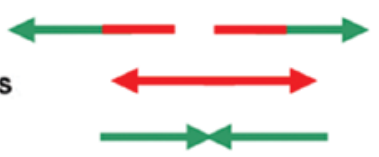

Mock

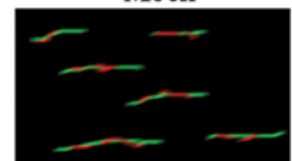

ING2-ANLS

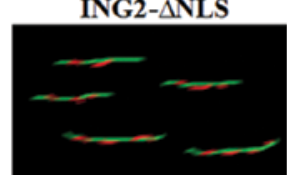

Figure 4. (A) Apoptotic ratios of cells transfected with various ING2 forms were determined using Annexin V/PI double-staining assays. (B) Cell cycle changes were determined by staining with PI. (C) Cells transfected with various ING2 forms were stained for SA- $\beta$-gal activity. (D) Representative images showing DNA fibers spread onto microscopic slides and immunostained with antibodies recognizing BrdU or IdU.
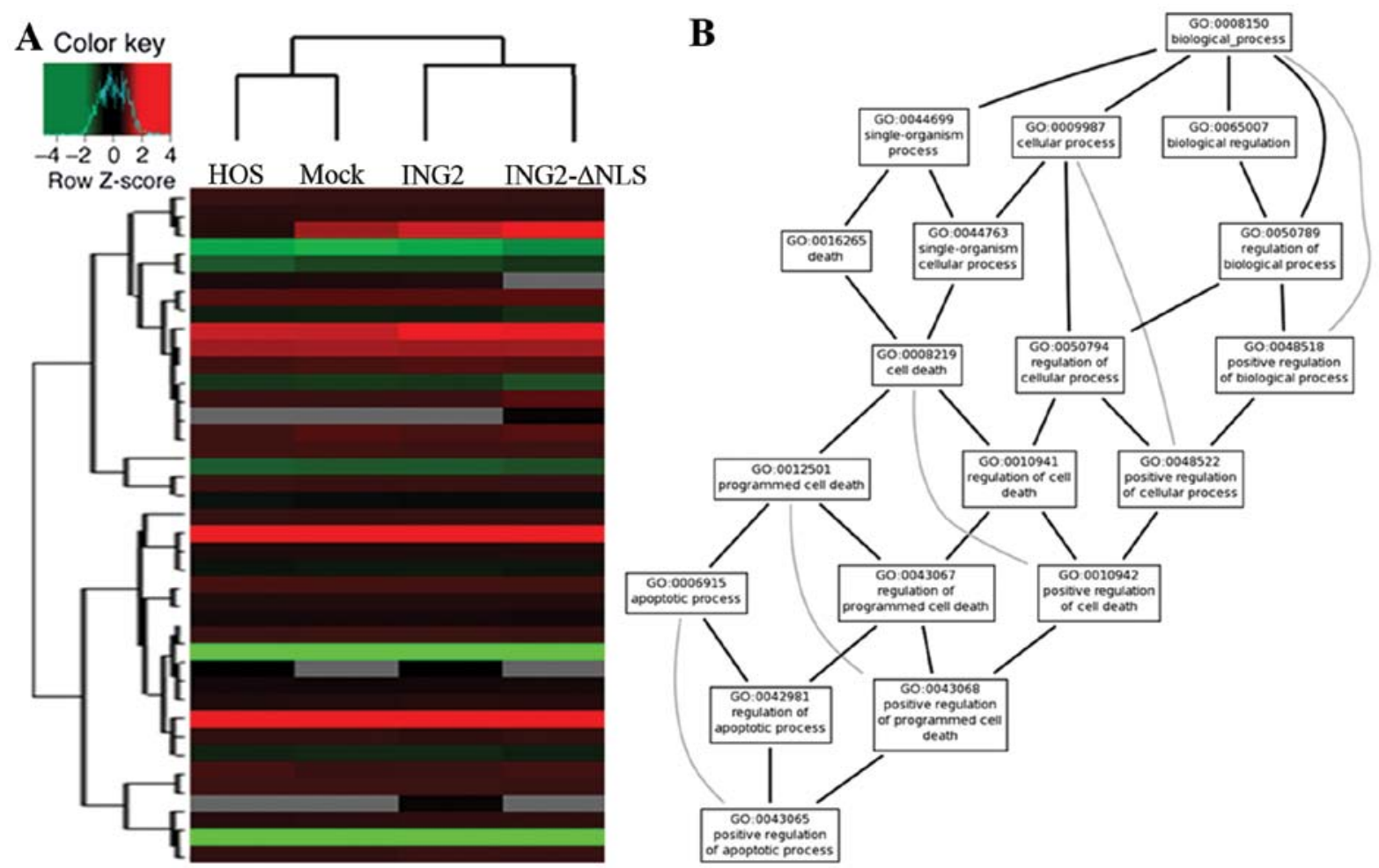

Figure 5. (A) Microarray analysis identified many subtle differences in RNA expression. The intensities are mean centered, with green indicating lower values and red indicating higher values. (B) The canonical signaling pathways of ING2 were generated using Gene Ontology (GO) software (http://www. geneontology.org). 
cytoplasmic ING2 protein. As shown in Fig. 3, the biochemical and the structural characteristics of the ING2 protein without NLS was similar to the intact ING2 protein. Secondly, we confirmed that nuclear ING2 protein induced apoptosis, G1 phase arrest and senescence in the osteosarcoma cells. Previous research also showed similar functions for ING2. For example, ING2 overexpression in young fibroblasts was found to arrest cells in the G1 phase and induce senescence (22). The PHD finger of ING2 has been implicated in the negative regulation of cell proliferation through modulation of p53 acetylation in response to DNA damage (23). Finally, we detected the mechanism of ING2 in osteosarcoma cells using Affymetrix GeneChip analysis. Previous reports have shown that ING2 interacts with HDAC1 and mSin3A, and has the potential ability to bind to $\mathrm{H} 3 \mathrm{~K} 4 \mathrm{me} 3(5,24)$. Other studies also found that ING2 interacts with the p300 acetyltransferase to enhance p53 acetylation on lysine 382 to increase transcription of p53 target genes, particularly p21 and Bax to control cell cycle arrest, senescence and apoptosis $(6,22)$. Unfortunately, we did not identify any new mechanism of ING2 in the present study.

Overall, this study provides novel biochemical, structural and functional data on ING2 in osteosarcoma cells. These results thus reinforce the role of ING2 as a tumor-suppressor gene, acting by controlling cell cycle progression and cell proliferation.

\section{Acknowledgements}

We are indebted to Mr. Xin-Yu Zhang for the pcDNA3.1-ING2 and pcDNA3.1-ING2- $\Delta$ NLS plasmids.

\section{References}

1. Bielack SS, Kempf-Bielack B, Delling G, et al: Prognostic factors in high-grade osteosarcoma of the extremities or trunk: an analysis of 1,702 patients treated on Neoadjuvant Cooperative Osteosarcoma Study Group protocols. J Clin Oncol 20: 776-790, 2002.

2. Meyers PA and Gorlick R: Osteosarcoma. Pediatr Clin North Am 44: 973-989, 1997.

3. Dai X, Ma W, He X and Jha RK: Review of therapeutic strategies for osteosarcoma, chondrosarcoma, and Ewing's sarcoma. Med Sci Monit 17: RA177-RHA190, 2011.

4. Watson RL, Spalding AC, Zielske SP, et al: GSK3beta and betacatenin modulate radiation cytotoxicity in pancreatic cancer. Neoplasia 12: 357-365, 2010.

5. Shi X, Hong T, Walter KL, et al: ING2 PHD domain links histone $\mathrm{H} 3$ lysine 4 methylation to active gene repression. Nature 442 : 96-99, 2006
6. Nagashima M, Shiseki M, Miura K, et al: DNA damage-inducible gene p33ING2 negatively regulates cell proliferation through acetylation of p53. Proc Natl Acad Sci USA 98: 9671-9676, 2001.

7. Nagashima M, Shiseki M, Pedeux RM, et al: A novel PHDfinger motif protein, p47ING3, modulates p53-mediated transcription, cell cycle control and apoptosis. Oncogene 22: 343-350, 2003.

8. Shiseki M, Nagashima M, Pedeux RM, et al: p29ING4 and p28ING5 bind to p53 and p300 and enhance p53 activity. Cancer Res 63: 2373-2378, 2003.

9. Guérillon C, Larrieu D and Pedeux R: ING1 and ING2: multifaceted tumor suppressor genes. Cell Mol Life Sci 70: 3753-3772, 2013.

10. Borkosky SS, Gunduz M, Nagatsuka H, et al: Frequent deletion of ING2 locus at 4q35.1 associates with advanced tumor stage in head and neck squamous cell carcinoma. J Cancer Res Clin Oncol 135: 703-713, 2009.

11. Zhang HK, Pan K, Wang H, et al: Decreased expression of ING2 gene and its clinicopathological significance in hepatocellular carcinoma. Cancer Lett 261: 183-192, 2008.

12. Kumamoto K, Fujita K, Kurotani R, et al: ING2 is upregulated in colon cancer and increases invasion by enhanced MMP13 expression. Int J Cancer 125: 1306-1315, 2009.

13. Kyte J and Doolittle RF: A simple method for displaying the hydrophobic character of a protein. J Mol Biol 157: 105-132, 1982.

14. Hopp TP and Woods KR: Prediction of protein antigenic determinants from amino acid sequences. Proc Natl Acad Sci USA 78: 3824-3828, 1981

15. Vihinen M, Torkkila E and Riikonen P: Accuracy of protein flexibility predictions. Proteins 19: 141-149, 1994.

16. Trier NH, Hansen PR and Houen G: Production and characterization of peptide antibodies. Methods 56: 136-144, 2012.

17. Dimri GP, Lee X, Basile G, et al: A biomarker that identifies senescent human cells in culture and in aging skin in vivo. Proc Natl Acad Sci USA 92: 9363-9367, 1995.

18. Jackson DA and Pombo A: Replicon clusters are stable units of chromosome structure: evidence that nuclear organization contributes to the efficient activation and propagation of $\mathrm{S}$ phase in human cells. J Cell Biol 140: 1285-1295, 1998.

19. Walzak AA, Veldhoen N, Feng X, Riabowol K and Helbing CC. Expression profiles of mRNA transcript variants encoding the human inhibitor of growth tumor suppressor gene family in normal and neoplastic tissues. Exp Cell Res 314: 273-285, 2008.

20. Ythier D, Brambilla E, Binet R, et al: Expression of candidate tumor suppressor gene ING2 is lost in non-small cell lung carcinoma. Lung Cancer 69: 180-186, 2010.

21. Lu F, Dai DL, Martinka M, Ho V and Li G: Nuclear ING2 expression is reduced in human cutaneous melanomas. $\mathrm{Br} \mathbf{J}$ Cancer 95: 80-86, 2006.

22. Pedeux R, Sengupta S, Shen JC, et al: ING2 regulates the onset of replicative senescence by induction of p300-dependent p53 acetylation. Mol Cell Biol 25: 6639-6648, 2005.

23. Gozani O, Karuman P, Jones DR, et al: The PHD finger of the chromatin-associated protein ING2 functions as a nuclear phosphoinositide receptor. Cell 114: 99-111, 2003.

24. Pena PV, Davrazou F, Shi X, et al: Molecular mechanism of histone H3K4me3 recognition by plant homeodomain of ING2. Nature 442: 100-103, 2006. 